\title{
High Accurate Howland Current Source: Output Constraints Analysis
}

\author{
Pedro Bertemes-Filho", Alexandre Felipe, Volney C. Vincence \\ Department of Electrical Engineering, State University of Santa Catarina (UDESC), Joinville, Brazil \\ Email: " bertemes@joinville.udesc.br
}

Received July 24, 2013; revised August 24, 2013; accepted August 31, 2013

Copyright (C) 2013 Pedro Bertemes-Filho et al. This is an open access article distributed under the Creative Commons Attribution License, which permits unrestricted use, distribution, and reproduction in any medium, provided the original work is properly cited.

\begin{abstract}
Howland circuits have been widely used as powerful source for exciting tissue over a wide frequency range. When a Howland source is designed, the components are chosen so that the designed source has the desired characteristics. However, the operational amplifier limitations and resistor tolerances cause undesired behaviors. This work proposes to take into account the influence of the random distribution of the resistors in the modified Howland circuit over the frequency range of $10 \mathrm{~Hz}$ to $10 \mathrm{MHz}$. Both output current and impedance of the circuit are deduced either considering or the operational amplifiers parameters. The probability density function due to small changes in the resistors of the circuit was calculated by using the analytical modeling. Results showed that both output current and impedance are very sensitive to the resistors variations. In order to get higher output impedances, high operational amplifier gains are required. The operational amplifier open-loop gain increases as increasing the sensitivity of the output impedance. The analysis done in this work can be used as a powerful co-adjuvant tool when projecting this type of circuit in Spice simulators. This might improve the implementations of practical current sources used in electrical bioimpedance.
\end{abstract}

Keywords: Howland Current Source; Electrical Bioimpedance; Probability Density Function; Resistors Mismatching

\section{Introduction}

Because of its simplicity, stability and other advantages, the voltage controlled current source (VCCS) has been widely used in many applications, such as in neuron-stimulation systems [1-3], single-electrode capacitive sensors [4], electrical impedance tomography (EIT) systems both for industrial and medical applications [5,6] and bioimpedance analysis (BIA) for tissue characterization [7,8]. It is also been used for exciting tissue for cancer characterization in electrical impedance spectroscopy [9-11]. Most VCCS circuits in BIA use the Howland current source (HCS) [12]. The first HCS circuit was proposed by Howland in 1962 [13] for converting a voltage into a current. However, it suffers from output voltage compliance [7]. Therefore, the modified version of the circuit has been proposed and widely used $[14,15]$.

The most important requirement in EIS systems is to assure that the injecting current, also called source current, has constant amplitude over a wide frequency range, which should be obtained by a high output impedance circuit [7]. However, stray capacitances [4] and non-

${ }^{*}$ Corresponding author. idealities of the operational amplifiers used for the design [15] reduce the current amplitude and introduce phase shift errors at higher frequencies. However, some of these requirements are essentially in conflict with each other. For example, the frequency of the current source is practically limited to $100 \mathrm{kHz}$ when the output impedance is required to be sufficiently larger, say larger than 1 $\mathrm{M} \Omega$ [1]. In most publications, the analysis of the circuit is based on either simplified ideal opamp circuits or using simulation tools, e.g. PSpice or Multisim from NI $[12,16]$. In many cases, however, the formulas they used are not suitable because the calculation errors are too large with a high-frequency current source [16]. Furthermore, the formulas do not take into account the mismatch between the resistors used to design such a current source. The HCS circuit is sensitive upon this mismatching [7].

The objective of this work is to investigate the probability density function for the analysis of the Howland circuit. It also investigates the sensibility of the Howland current source over the frequency range of $10 \mathrm{~Hz}$ to 10 $\mathrm{MHz}$ due to value mismatching between resistors. 


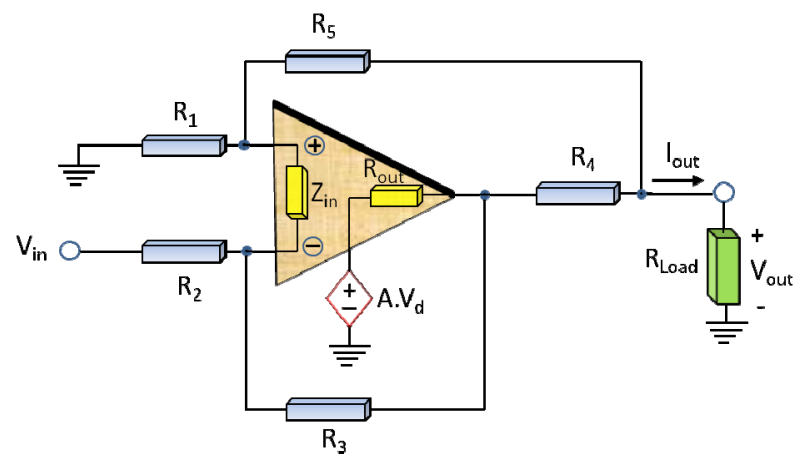

Figure 1. Schematic diagram of the current source with grounded load [17], where $V_{d}$ is the differential voltage across the input terminals.

\section{Howland Current Source}

Figure 1 shows the modified Howland current source (HCS) circuit used for modeling the output characteristics taking into account the effects of the resistors tolerances. If all resistors are perfectly matched and the operational amplifier (opamp) has got a large gain, then the output current is given by $-V_{\text {in }} / r$ (assuming $R_{2}=R_{3}=R_{5}$ $=R, R_{4}=r$ and $R_{1}=R+r$ ). This approximation yields a good result if the Opamp gain is sufficiently high and the load impedance is small.

In order to get good Common Mode Rejection Ratio (CMRR) and high output impedance it is necessary to trim the resistors and choose FET inputs amplifiers. On the other hand, the non-ideal characteristics of the operational amplifiers (i.e., input impedance $Z_{i n}$, output resistance $R_{o}$ and open-loop gain $A$ ) reduce frequency bandwidth of both output current and impedance.

\subsection{Circuit Nodal Equations}

The circuit can be characterized by the nodal Equations (1)-(4), where $V_{1}$ is the voltage at non-inverting input of the Opamp, $V_{2}$ is the inverting input of the Opamp and $V_{3}$ is the voltage at the output of the Opamp. After determining the voltages the output current is calculated by Equation (4). The transconductance gain is calculated by $I_{\text {out }} / V_{\text {in }}$ with Vout grounded. On the other hand, the output impedance $Z_{\text {out }}$ is calculated by $I_{\text {out }} / V_{\text {out }}$ with $V_{\text {in }}$ grounded.

$$
\begin{gathered}
\frac{V_{1}-V_{\text {out }}}{R_{5}}+\frac{V_{1}}{R_{1}}+\frac{V_{1}-V_{2}}{Z_{\text {in }}}=0 \\
\frac{V_{2}-V_{\text {in }}}{R_{2}}+\frac{V_{2}-V_{1}}{Z_{\text {in }}}+\frac{V_{2}-V_{3}}{R_{3}}=0 \\
\frac{V_{3}-V_{2}}{R_{3}}+\frac{V_{3}-V_{\text {out }}}{R_{4}}+\frac{V_{3}-\left(V_{1}-V_{2}\right) A}{R_{o}}=0
\end{gathered}
$$

$$
\begin{gathered}
I_{\text {out }}=\frac{V_{3}-V_{\text {out }}}{R_{4}}+\frac{V_{1}-V_{\text {out }}}{R_{5}} \\
A=A(j \omega)=\frac{A_{o}}{1+j \omega / \omega_{c o}}
\end{gathered}
$$

where $A_{o}$ and $\omega_{c o}$ are the open-loop gain modulus at zero frequency and the open-loop gain corner frequency, respectively.

$$
Z_{\text {in }}(j \omega)=\frac{1}{1 / R_{\text {in }}+j \omega C_{\text {in }}}
$$

where $R_{\text {in }}$ and $C_{\text {in }}$ are both input resistance and capacitance of the opamp, respectively.

The Norton theorem can be applied to the circuit shown in Figure 1 then the shunt impedance $Z_{N}$ of the circuit can be calculated by Equation (7) and the transconductance gain $G_{N}$ can be calculated by Equation (8).

$$
\begin{aligned}
& Z_{N}=\left.\left(-I_{\text {out }} / V_{\text {out }}\right)\right|_{V_{\text {in }}=0} \\
& G_{N}=\left.\left(I_{\text {out }} / V_{\text {in }}\right)\right|_{V_{\text {out }}=0}
\end{aligned}
$$

\section{Probability Distribution Function}

The probability distribution functions (PDF) of the source parameters are calculated by classifying and counting the elements falling in each class. It is expected that any combination of resistors of $\pm 1 \%$ tolerance $(\delta)$ may produce either significant reduction in the output impedance or a large variation in the transconductance gain $\left(I_{\text {out }} / V_{\text {in }}\right)$.

The box below shows the pseudo-code program developed in a very high level for the determination of some statistics of the circuit, which was projected by using resistors whose values distribution are known.

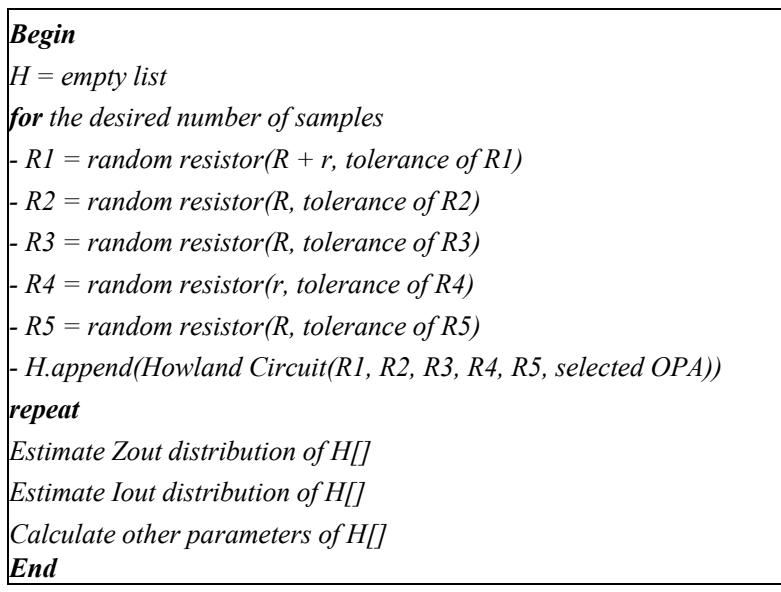

This program was implemented in $R$ scripting language, which is a language and environment for statistical computing and graphics. In this work the resistors 
were distributed uniformly by using the run if $R$ function, and the estimation of probability density functions was made with the density $R$ function. More detailed information can be seen in the link http://www.r-project.org.

A Howland current source is designed by using $R=$ $100 \mathrm{k} \Omega, r=1 \mathrm{k} \Omega$ and it is assumed that each resistor has a tolerance $\delta$ of $\pm 1 \%$ with no correlation to each other.

It was investigated the effect of the tolerance of the resistors in the transconductance gain when designing a Howland current source, Equation (4) was used by assuming a tolerance of $\pm 1 \%$ for each discrete frequency over the range $10 \mathrm{~Hz}$ to $1 \mathrm{MHz}$. It was used the operational amplifier OPA655 from Texas Instruments, where the technical specifications are shown in Table 1.

The result is shown in Figure 2, where the probability density is represented by a gray scale. The white points represent the values where the transconductance might not be achieved at that frequency. It can also be seen that the frequency dependency mater is much more relevant than the tolerance of the resistors.

The transconductance gain $G_{N}$ can be approximately calculated by the ratio $-R_{3} /\left(R_{2} R_{4}\right)$, thus if the tolerance of the resistors are equal to $\delta$, the tolerance of the transconductance gain will have a tolerance of approximately $3 \delta$. It implies that the use of a wideband operational amplifier will produce a very stable transconductance gain. On the other hand, the output impedance $Z_{\text {out }}$ will be significantly changed by the tolerance of the resistors.

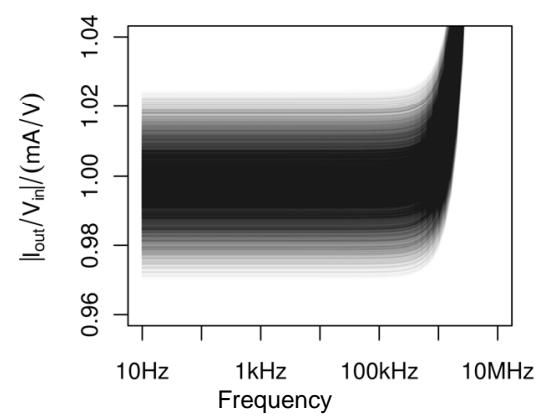

Figure 2. Probability distribution of the transconductance gain, using the OPA655 and resistors of $\pm 1 \%$ tolerance.

Table 1. The main technical specifications of the operational amplifiers used in this work.

\begin{tabular}{cccccc}
\hline & $\boldsymbol{R}_{\text {in }}(\mathbf{M} \boldsymbol{\Omega})$ & $\boldsymbol{C}_{\text {in }}(\mathbf{p F})$ & $\boldsymbol{R}_{\mathbf{0}}(\boldsymbol{\Omega})$ & $\boldsymbol{A}_{\mathbf{0}}(\mathbf{d B})$ & $\boldsymbol{\omega}_{\boldsymbol{c 0}}(\boldsymbol{\pi H z})$ \\
\hline OPA657 & 1000 & 0.7 & 0.02 & 75 & 600,000 \\
OPA656 & 1000 & 0.7 & 0.01 & 66 & 200,000 \\
OPA655 & 1000 & 1.2 & 0.04 & 58 & 400,000 \\
LMH6654 & 4 & 1.8 & 0.08 & 80 & 50,000 \\
OP07C & 50 & NF & 60 & 115 & 4 \\
TL081 & 1000 & 3.0 & 10 & 105 & 50 \\
uA741 & 2 & 1.4 & 75 & 97 & 30 \\
\hline
\end{tabular}

Note that $A_{0}$ of the opamp has been extracted from the plots of the open-loop gain versus frequency presented in the datasheet.

\section{Output Impedance Modeling}

\subsection{Model 1: Infinite Open-Loop Gain}

By solving Equation (4) and consider a suficiently high open-loop gain $A$, the output impedance can be briefly calculated by Equation (9)

$$
\left.Z_{\text {out }}\right|_{A=\infty}=\frac{R_{2} R_{4} R_{5}+R_{1} R_{2} R_{4}}{R_{2} R_{5}+R_{2} R_{4}-R_{1} R_{3}}
$$

It can be seen in Equation (9) that the relative error of the numerator is approximately theree times larger than the resistor tolerances. The denominator of the equation would be zero if the resistors were perfectly matched. Therefore, the output would be very dependent on their values. By assuming equal tolerances for all resistors with a fraction nominal value of each resitor, and also using an operational amplifier with high open-loop gain $A$, the minimum ouput impedance can be calculated by Equation (10).

$$
Z_{\text {out, } \min }(\delta)=\frac{r R}{2 \delta(R+r)}
$$

where $R=R_{2}=R_{3}=R_{5}, r=R_{4}$ and $R+r=R_{1}$.

It can be seen in Equation (10) that if $R$ is much greater than $r$ and assuming equal tolerances for all resistors, then variations on the resistor $R_{4}(=r)$ become much less signicant for the output impedance compared to the variations on other resistors.

\subsection{Model 2: Finite Open-Loop Gain}

By sampling randomly the resistors to fall next to theirs nominal values and calculating $Z_{\text {out }}$, it would imply that we are assigning to $Z_{\text {out }}$ randomly values which are selected near by a pole of a function. It means that high values can be obtained as well as the small ones, which, in turns, are much more difficult to happen. Furthermore, as it can be seen in Figure 3, the output impedance has a finite upper limit.

Both $V_{\text {out }}$ and $I_{\text {out }}$ can be written by two polynomial functions, which are both related to $A, Z_{\text {in }}, R_{o}$ and the resistors. Considering $Z_{\text {in }}$ sufficiently large and $R_{o}$ sufficiently small, $Z_{\text {out }}$ can be calculated according to Equation (11) as a function of the open-loop gain $A$.

$$
Z_{\text {out }}(A)=\frac{R_{2} R_{E 1} A+R_{E 1}\left(R_{2}+R_{3}\right)}{A\left(R_{E 2}-R_{1} R_{3}\right)+\left(R_{2}+R_{3}\right)\left(R_{1}+R_{E 2} / R_{2}\right)}
$$

where $R_{E 2}=R_{2}\left(R_{4}+R_{5}\right)$ and $R_{E 1}=R_{4}\left(R_{1}+R_{5}\right)$.

Perturbations in the coefficients of the gain $A$ in both numerator and denominator of Equation (11) may lead it to negative values and then to instability. Therefore, they are much more important than the independent terms. The numerator can be approximately bounded by $r(A+2)$ $R(2 R+r)(1 \pm 3 \delta)$, by assuming $R=R_{2}=R_{3}=R_{5}, r=R_{4}$ and $R+r=R_{1}$. On the other hand, the coefficient of the 


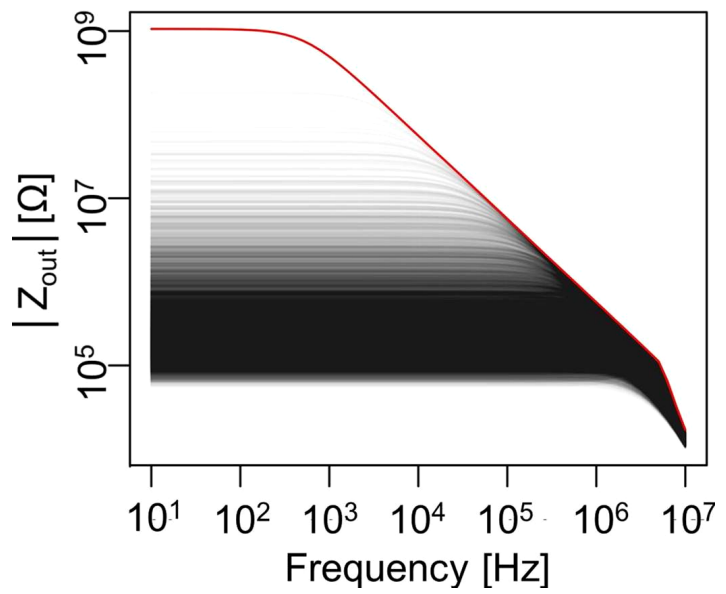

Figure 3. Probability distribution of the output impedance, using the OPA655 and resistor tolerances of $\pm 1 \%$.

gain $A$ in the denominator of Equation (11) may assume both positive and negative values in the range of $-2 \delta R(R$ $+r)$ to $+2 \delta R(R+r)$, and the independent term is $4 R(R+$ $r)(1+2 \delta)$.

By considering small perturbations, $Z_{\text {out }}$ can be approximated by $A /\left(a_{1} A+a_{0}\right)$, where the denominator of this expression in the complex plane is a straight line crossing $a_{0}$ with an angle $\theta(A)$. Therefore, it can be calculated that the modulus of $Z_{\text {out }}$ is inversely proportional to the distance from a point in that line to the origin and the phase is the difference between the arguments of such a point and the gain $A$. As a result, the modulus of $Z_{\text {out }}$ can be written as a function of its phase, as shown in Equation (12).

$$
\left|Z_{\text {out }}\right| \cong \frac{r R(r+2 R)}{a_{1}+a_{0} / A}
$$

where

$$
\begin{array}{r}
a_{0}=2 R(r+R)\left[2+\frac{r+2 R}{Z_{\text {in }}}\right] \\
a_{1}=R_{2}\left(R_{5}+R_{4}\right)-R_{1} R_{3} \\
|A|=A_{0} \cdot \cos (\theta(A))
\end{array}
$$

By doing a simple geometric analysis in Equation (12), it can be observed that the distribution of $Z_{\text {out }}$ in the complex plane results into a circle centered at the imaginary axis with one of its vertices at the origin. It can be concluded that small $Z_{\text {out }}$ has a phase near zero or -180 degrees, as the resistors are combined to give a higher $Z_{\text {out }}$ and then the phase tends to -90 degrees, as shown in Equation (16) by assuming that the Equation (17) is satisfied.

$$
\left|Z_{\text {out,max }}\right|=\frac{r R(r+2 R)}{\left|\operatorname{Im}\left(a_{0} / A\right)\right|}
$$

$$
R_{2}\left(R_{5}+R_{4}\right)-R_{1} R_{3}+\operatorname{Re}\left(a_{0} / A\right)=0
$$

By considering negligible variations in the denominator of Equation (12) and also assuming only real values for the coefficient $a_{0}$ (see Equation 13), the $Z_{\text {out }}$ phase can be given by " $-\theta\left(a_{1}+a_{0} / A\right)$ " and the modulus by " $r R(2 R$ $+r) /\left|a_{1}+a_{0}\right|$ ". The term " $a_{1}+a_{0} / A$ " represents a horizontal line in the complex plane, so that the product between " $\left|a_{1}+a_{0} / A\right|$ " and " $\sin \left[\theta\left(a_{1}+a_{0} / A\right)\right]$ " is always constant. Therefore, $Z_{\text {out }}$ can be approximated by a circumference of radius $\left|Z_{\text {out,max }}\right| / 2$ according to Equation (18).

$$
\left|Z_{\text {out }}\right|=\left|Z_{\text {out,max }}\right| \sin \left[-\theta\left(Z_{\text {out }}\right)\right]
$$

Figure 4 shows both real and imaginary parts of $Z_{\text {out }}$ calculated by using a set of random resistors $(r=1 \mathrm{k} \Omega \pm$ $0.1 \%$ and $R=100 \mathrm{k} \Omega \pm 0.1 \%$ ) and the operational amplifiers TL081 (graphics 1, 2 and 3), uA741 (graphics 4, 5 and 6) and OPA655 (graphics 7,8 and 9), according to the parameters shown in Table 1. Simulations were made with and without the output resistance $R_{0}$. The red points were calculated values by using randomly values for the resistors in the range $+0.1 \%$ and $-0.1 \%$. The blue circle represents the geometric space in the complex plan which contains the calculated points according to Equation 12. The simulations show the results at three different frequencies: $10 \mathrm{KHz}$ (first column); $100 \mathrm{kHz}$ (second column); and $1 \mathrm{MHz}$ (third column). It can be seen that the MMHCS circuit designed by using the OPA655 has the highest output impedance, which is approximately $1.02 \mathrm{M} \Omega$ at $10 \mathrm{kHz}$. It can also be seen that the probability of getting the maximum output impedance does depend on the frequency and opamp used which, in turns, depends on the open-loop gain corner frequency. The graphic 6 shows that $Z_{\text {out }}$ is equal to zero at $1 \mathrm{MHz}$ when using the opamp uA741.

Figure 5 shows the output impedance of the MMHCS designed by the operational amplifiers TL081 (first column) and OPA655 (second column) at $10 \mathrm{kHz}$, using resistor tolerances of $0.1 \%$ (graphics "a" and "b"), $1 \%$ (graphics "c" and "d") and 10\% (graphics "e" and "f"). It can be calculated that the maximum $Z_{\text {out }}$ is approximately $213.6 \mathrm{k} \Omega$ when using the opamp TL081 whereas 1.06 $\mathrm{M} \Omega$ for the OPA655 one, considering resistor tolerances of $0.1 \%$. However, the probability of getting such a value is much smaller for the OPA655 case. It can also be seen that the probability density on getting maximum Zout increases significantly as increasing the resistor tolerances.

\section{Mirrored Howland Current Source: Modeling}

In order to improve the stability of the circuit, two single-ended Howland circuits can be set together in order to have a differential output current, which is also called 

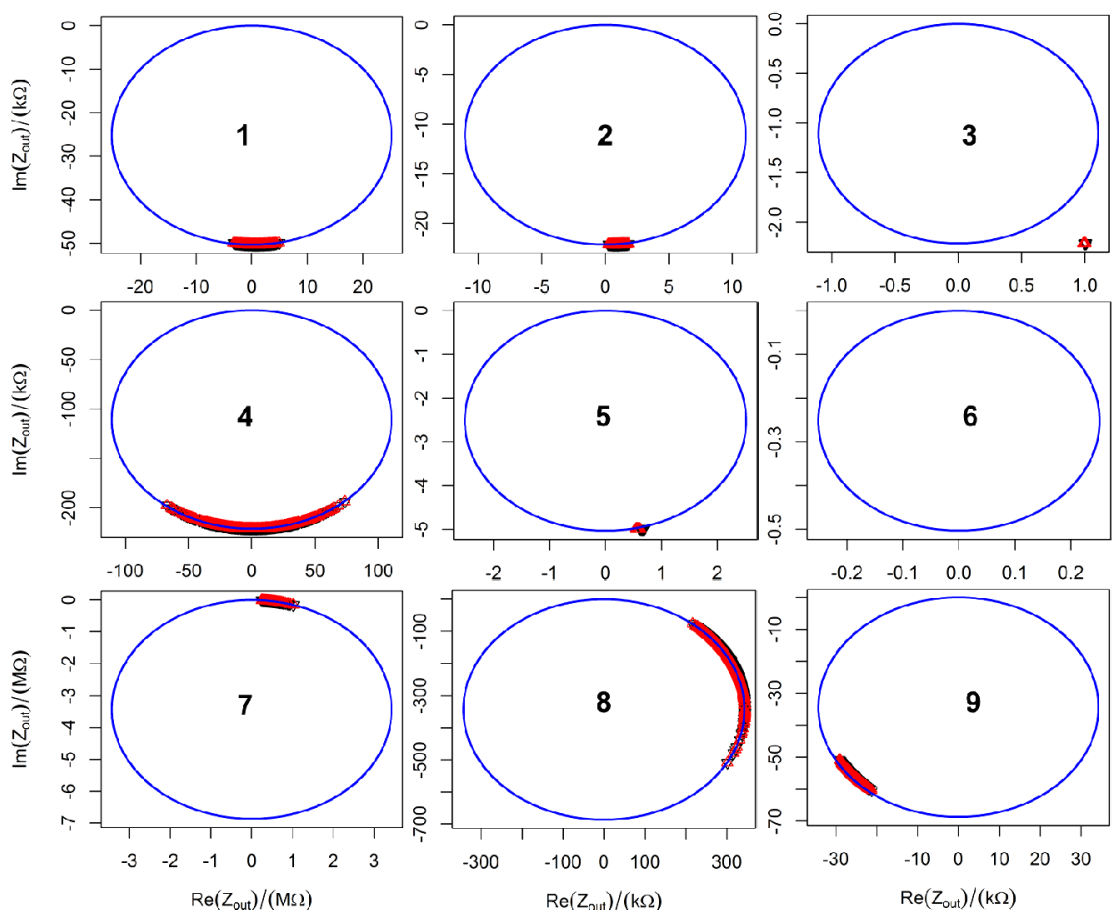

Figure 4. The real and imaginary part of the output impedance by using resistor tolerance of $0.1 \%$.
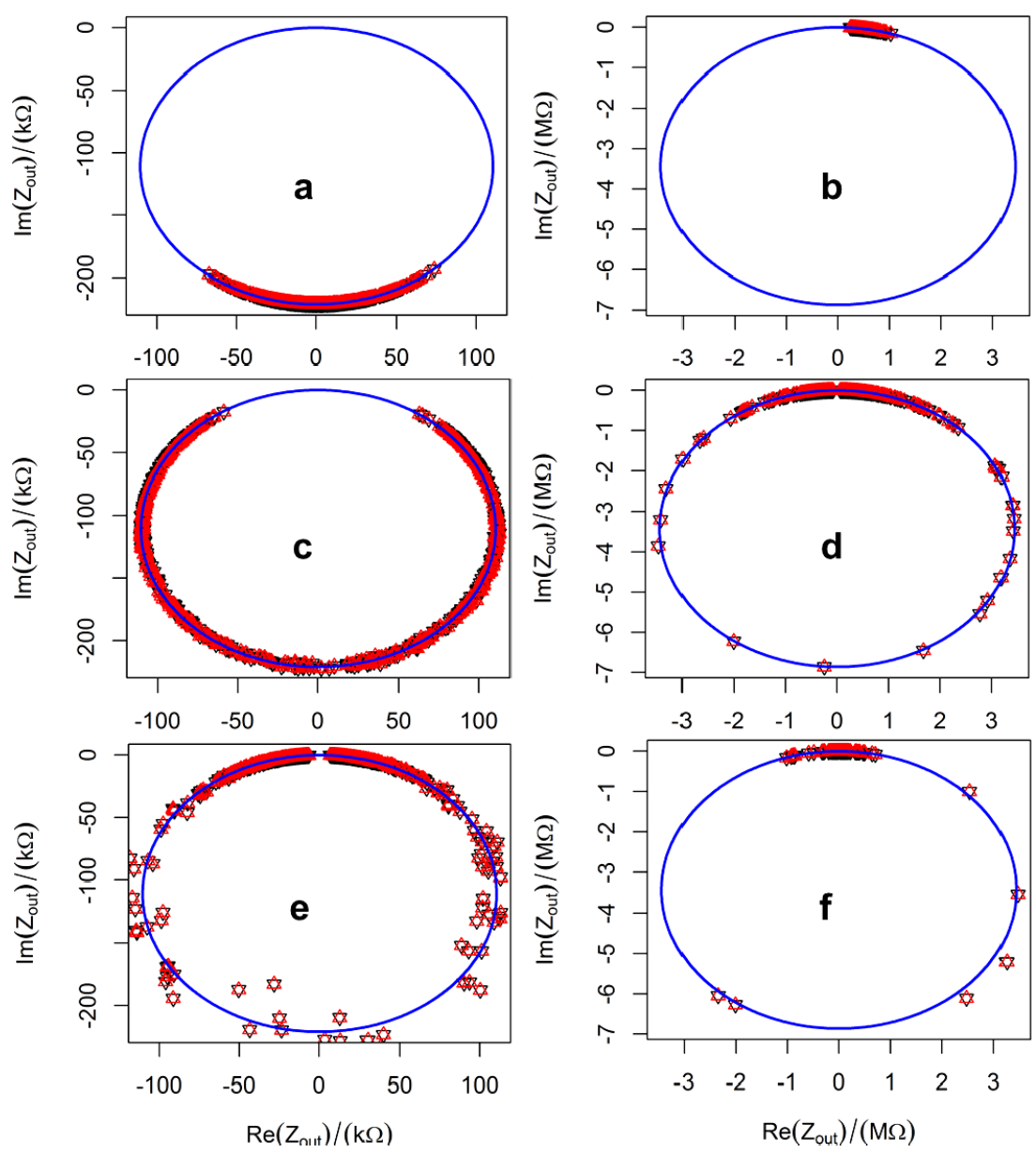

Figure 5. Changes in the output impedance at $10 \mathrm{kHz}$ by using different resistor tolerances. 
as Mirrored Modified Howland Current Source

(MMHCS) and it is shown in Figure 6 [17]. This type of current source uses two equal input voltages with 180 degrees phase shift from each other but also has a unique reference for both sides of the circuit, which, in turns, reduce the feed-through capacitance between output and input. This topology may significantly reduce the even harmonics of the output current, and then improving its linearity and reducing the amount of output voltage level at each side of this current source.

The MMHCS can be represented by its Norton equivalent circuit, as shown in Figure 7. The output voltage at each side of the load can then be calculated according to Equations (19) and (20), where $I_{N 1}$ and $I_{N 2}$ are the shortcircuit currents and $Z_{N 1}$ and $Z_{N 2}$ are the output impedance of each side of the circuit.

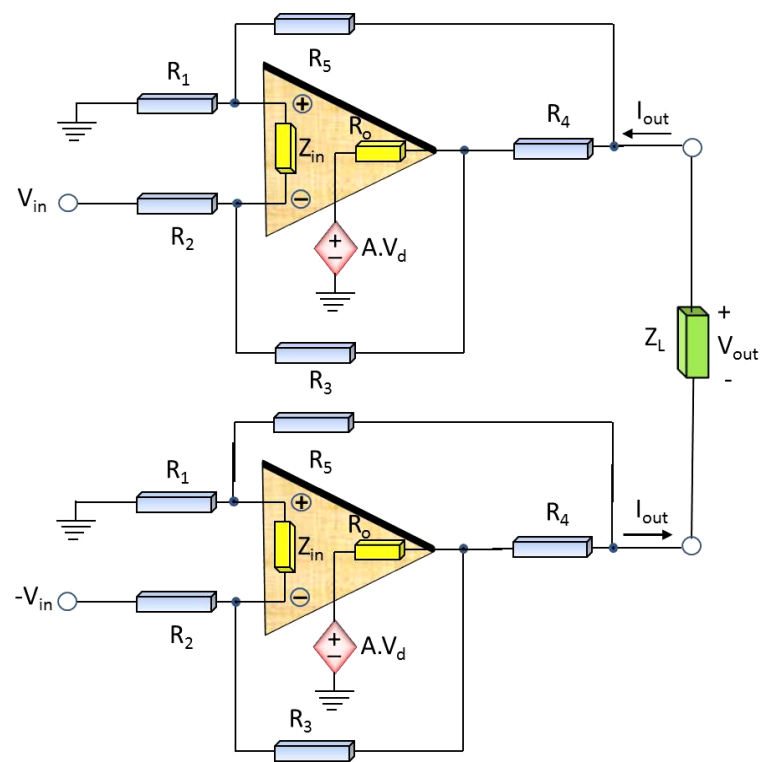

Figure 6. Schematic diagram of the mirrored modified Howland current source.

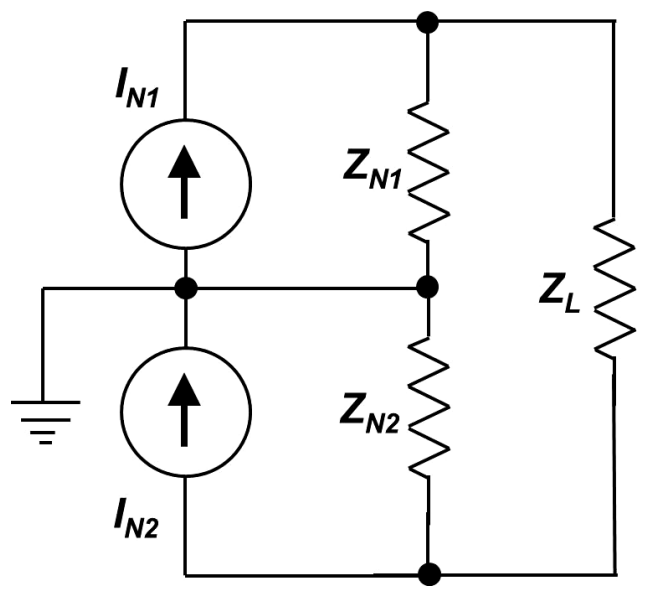

Figure 7. Equivalent Norton circuit for the mirrored modified Howland current source.

$$
\begin{aligned}
& V_{\text {out } 1}=\frac{I_{N 1} \cdot Z_{N 2} \cdot Z_{N 2}-I_{N 2} \cdot Z_{N 2} \cdot\left(Z_{L}+Z_{N 1}\right)}{Z_{L}+Z_{N 1}+Z_{N 2}} \\
& V_{\text {out } 2}=\frac{I_{N 1} \cdot Z_{N 1} \cdot\left(Z_{L}+Z_{N 2}\right)-I_{N 2} \cdot Z_{N 2} \cdot Z_{N 1}}{Z_{L}+Z_{N 1}+Z_{N 2}}
\end{aligned}
$$

The output current in the load impedance $Z_{L}$ can then be calculated according to Equation (21). As a result, the differential output voltage $V_{\text {diff }}$ can be calculated by Equation (22).

$$
\begin{array}{r}
I_{L}=\frac{I_{N 1} \cdot Z_{N 1}+I_{N 2} \cdot Z_{N 2}}{Z_{L}+Z_{N 1}+Z_{N 2}} \\
V_{\text {diff }}=\frac{\left(I_{N 1}-I_{N 2}\right) \cdot Z_{N 1} \cdot Z_{N 2}+\left(V_{N 1}-V_{N 2}\right) \cdot Z_{L} / 2}{Z_{L}+Z_{N 1}+Z_{N 2}}
\end{array}
$$

where $V_{N 1}=Z_{N 1} I_{N 1}$ and $V_{N 2}=Z_{N 2} I_{N 2}$.

It is important to note that if the output impedances $Z_{N 1}$ and $Z_{N 2}$ have got similar high values, then it is expected that the difference $Z_{N 1}-Z_{N 2}$ be very small whereas the $\operatorname{sum} Z_{N 1}+Z_{N 2}$ be very high. It can be seen in (22) that the differential voltage $V_{\text {diff }}$ reduces to the product between $\left(I_{N 1}-I_{N 2}\right)$ and $Z_{N 1,2}$, for example if the output impedances of the current source are hundred times larger than the load impedance. A difference of $1 \%$ in $I_{N 1}$ or $I_{N 2}$ can duplicate the voltage in one of the load terminals. The difference at the output which is seeing by each terminal leads to a common mode voltage, which is dependent of the load charge. This results from the fact that each side of the current source tries to fix the output current to a different value.

\section{Discussions}

\subsection{Output Impedance}

It is predicted by Equation (12) that if the open-loop gain $A$ is large, then it is very difficult to set the output impedance to its optimal value. It was showed that as decreasing the gain $A$ the size of the impedance circle is also decreased and then the region away from the origin become more statistically populated, as shown in Figure 3.

Researches have been using the differential (mirrored) output current sources for getting a more stable circuit and higher output impedance. The output impedance of this type of current source can be calculated by sum of the output impedances from both single-ended circuits (i.e. $Z_{N 1}+Z_{N 2}$ ). It is thought having only advantage reasons for doing that but it also suffers from high open-loop gain of the operational amplifiers. The real part of $Z_{\text {out }}$ can be either positive or negative and its imaginary part very small, then leading to a differential $Z_{\text {out }}$ even smaller than the single-ended output impedances. 


\subsection{Operational Amplifier Limitations}

As discussed before, from (22) it can be seen that small current differences at each side of the mirrored current source can increase the common mode voltage at the load terminals. In order to preserve those differences in the output current, the operational amplifiers have to supply the differential voltage as well as the common mode voltage.

The output impedance of the current source must be high otherwise the load current varies as varying the load impedance. By designing a high output impedance current source, the transconductance gains have to be precisely matched for preventing the operational amplifiers to work one against each other. This might be explained by the fact that they are connected in series, and then both circuits are designed to reject any variation in its output current.

\subsection{Tuning the Mirrored Current Source}

Tuning the mirrored Howland current source is not a simple task as it has to be done in both side of the circuit at the same time.

It was shown that the transconductance gain can be approximately given by the ratio between the resistor $-R_{3}$ and the product " $R_{2} R_{4}$ ". Consequently, it can be interpreted as a current gain, where instead of having an input voltage it has an input current $\left(=V_{\text {in }} / R_{2}\right)$. As a result, both branches of the circuit which contains the resistors $R_{2}$ (see Figure 6) should be connected in series in order to prevent input current differences at each side of the current source. Therefore, it can be used only one resistor $R_{2}$ for both source sides.

In order to have equal output characteristics at both sides of the mirrored modified Howland current source, both sides have to have an equal ratio of $R_{3} / R_{4}$. This might be solved by adding a potentiometer in series with one of $R_{4}$ for tuning the differential output current. In practice, this can be done by short-circuiting the output and measuring the output voltage while tuning the potentiometer to have a null output voltage. It might be necessary to connect a resistor at each side of the short-circuit in order to prevent saturation by the operational amplifiers.

By the fact that the resistor $R_{4}$ has a very small influence in the output impedance, this last can be tuned by varying either the resistor $R_{5}$ or $R_{1}$. The resistor $R_{1}$ of both sides can also be connected in series in order to reduce imbalances from these circuit sides, thus one of the resistors $R_{5}$ becomes the natural tuning element for controlling the output impedance of the mirrored modified Howland current source.

\subsection{Limitations}

The analytical analyses and the equations related to the output of the Howland current source have not considered any stray capacitances which might be found in practical circuits. Also, the numerical solutions for both output current and impedance of the circuit have not considered the common-mode input impedance of the operational amplifiers, as well as the common-mode rejection ratio (CMRR). It has to be pointed out that the probability density function was used by assuming that the resistor variations behave as a normal distribution. Therefore, care should be taken when analyzing the results obtained in this work, especially when they are related to practical circuits.

\section{Conclusions}

It was fully described the modeling of both output current and impedance of the Howland current source by considering the operational amplifier parameters and the mismatching between electrodes. It was shown that the output resistance of the operational amplifier does not play a role in the output characteristic of the Howland circuit whereas the open-loop gain causes a great impact on it.

It was also shown that the higher the gain, the most sensitive is the output impedance in respect to the resistors tolerances, as illustrated in Figures $\mathbf{4}$ and $\mathbf{5}$. In order to operate at high frequency, the open-loop gain of the opamp has to be very high. This explain why the resistors have to be precisely matched in order to obtain a MMHCS circuit which can deliver a constant differential output current into the load over a wide frequency range.

It can be concluded from this work that both side of the MMHCS should be very symmetrical if high output impedance from this type of circuit is desired. This is a very significant achievement found in this work for the design of high output impedance Howland current sources used in bioimpedance measurements. This may lead to empirical distribution of important characteristics of the MMCHS during the design and it might save time and money when this type of circuit is put in production scale. Furthermore, the error estimation provided here can guide researchers, who have no previous knowledge in Howland current source, to design a high quality circuit in terms of both output current and impedance spectra according to the application requirements.

\section{Acknowledgements}

This work was supported by the State University of Santa Catarina and the National Council for Scientific and Technological Development (grant 237931/2012-5).

\section{REFERENCES}

[1] P. Pouliquen, J. Vogelstein and R. Etienne-Cummings, 
"Practical Considerations for the Use of a Howland Current Source for Neuron-Stimulation," Proceedings of the IEEE Biomedical Circuits and Systems Conference, Baltimore, 20-22 November 2008, pp. 33-36.

http://dx.doi.org/10.1109/BIOCAS.2008.4696867

[2] E. Basham, Z. Yang and W. Liu, "Circuit and Coil DESIGN for in-Vitro Magnetic Neural Stimulation Systems," IEEE Transactions on Biomedical Circuits and Systems, Vol. 3, No. 5, 2009, pp. 321-331. http://dx.doi.org/10.1109/TBCAS.2009.2024927

[3] K. Sooksood, T. Stieglitz and M. Ortmanns, "An Active Approach for Charge Balancing in Functional Electrical Stimulation," IEEE Transactions on Biomedical Circuits and Systems, Vol. 4, No. 3, 2010, pp. 162-170. http://dx.doi.org/10.1109/TBCAS.2010.2040277

[4] D. X. Chen, X. Deng and W. Q. Yang, "Comparison of Three Current Sources for Single-Electrode Capacitance Measurement," Review of Scientific Instruments, Vol. 81, No. 3, 2010, pp. 1-3. http://dx.doi.org/10.1063/1.3367879

[5] R. A. Pease, "A Comprehensive Study of the Howland Current Pump," 2008. http://www.ti.com/lit/an/snoa474a/snoa474a.pdf

[6] J. Frounchi, F. Dehkhoda and M. H. Zarifi, "A Low-Distortion Wideband Integrated Current Source for Tomography Applications," European Journal of Scientific Research, Vol. 27, No. 1, 2009, pp. 56-65.

[7] P. Bertemes-Filho, "Tissue Characterization Using an Impedance Spectroscopy Probe," Ph.D. Thesis, University of Sheffield, Sheffield, 2002.

[8] S. Grimnes and O. G. Martinsen, "Bioimpedance and Bioelectricity Basics," 2nd Edition, Elsevier Ltd, Amsterdam, 2008.

[9] A. Keshtkar, Z. Salehnia and B. Shokouhi, "Bladder Cancer Detection Using Electrical Impedance Technique (Tabriz Mark 1)," Pathology Research International, Vol. 2012, 2012, pp. 1-5.

http://dx.doi.org/10.1155/2012/470101

[10] P. Aberg, I. Nicander and S. Ollmar, "Minimally Invasive Electrical Impedance Spectroscopy of Skin Exemplified by Skin Cancer Assessments," Proceeding of the 25th Annual International Conference of the IEEE Engineering in Medicine and Biology Society, Cancun, 17-21 September
2003, pp. 3211-3214

http://dx.doi.org/10.1109/IEMBS.2003.1280826

[11] P. Åberg, I. Nicander, J. Hansson, P. Geladi, U. Holmgren and S. Ollmar, "Skin Cancer Identification Using Multifrequency Electrical Impedance-A Potential Screening Tool," IEEE Transactions on Biomedical Engineering, Vol. 51, No. 12, 2004, pp. 2097-2102. http://dx.doi.org/10.1109/TBME.2004.836523

[12] D. H. Sheingold, "Impedance \& Admittance Transformations Using Operational Amplifiers," Lightning Empiricist, Vol. 12, No. 1, 1964, pp. 1-8.

http://www.philbrickarchive.org/1964-1_v12_no1_the_lightning_empiricist.htm

[13] P. Bertemes-Filho, R. G. Lima, M. B. P. Amato and H. Tanaka, "Performance of an Adaptative Multiplexed Current Source Used in Electrical Impedance Tomography," Proceeding of the 20th Brazilian Congress on Biomedical Engineering, São Pedro, 22-26 October 2006, pp. 11671170 .

[14] F. Seoane, R. Bragós and K. Lindecranz, "Current Source for Multifrequency Broadband Electrical Bioimpedance Spectroscopy Systems. A Novel Approach," Proceedings of the 28th Annual International Conference of the IEEE Engineering in Medicine and Biology Society, New York, 31 August-3 September 2006, pp. 5121-5125. http://dx.doi.org/10.1109/IEMBS.2006.259566

[15] F Seoane, R Macías, R Bragos and K Lindecrantz, "Simple Voltage-Controlled Current Source for Wideband Electrical Bioimpedance Spectroscopy: Circuit Dependences and Limitations," Measurement Science and Technology, Vol. 22, No. 11, 2011, pp. 1-11. http://dx.doi.org/10.1088/0957-0233/22/11/115801

[16] P. Bertemes-Filho, B. H. Brown and A. J. Wilson, "A Comparison of Modified Howland Circuits as Current Generators with Current Mirror Type Circuits," Physiological Measurement, Vol. 21, No. 1, 2000, pp. 1-6. http://dx.doi.org/10.1088/0967-3334/21/1/301

[17] P. Bertemes-Filho, L. H. Negri, A. Felipe and V. C. Vincence, "Mirrored Modified Howland Circuit for Bioimpedance Applications: Analytical Analysis," Journal of Physics: Conference Series, Vol. 407, No. 1, 2012, pp. 18. http://dx.doi.org/10.1088/1742-6596/407/1/012030 